Les ANNALES Les Annales de droit

DE DROIT

$8 \mid 2014$

Varia

\title{
Jugements de valeur et argumentation juridique
}

Value judgments and legal arguments

\section{Ángeles Ródenas Calatayud}

Traducteur : Guillaume Tusseau

\section{OpenEdition}

Journals

Édition électronique

URL : http://journals.openedition.org/add/753

DOI : 10.4000/add.753

ISSN : 2606-1988

\section{Éditeur}

Presses universitaires de Rouen et du Havre

\section{Édition imprimée}

Date de publication : 1 mai 2014

Pagination : 229-250

ISBN : 979-10-240-0161-6

ISSN : 1955-0855

\section{Référence électronique}

Ángeles Ródenas Calatayud, " Jugements de valeur et argumentation juridique », Les Annales de droit [En ligne], 8| 2014, mis en ligne le 14 mars 2018, consulté le 17 septembre 2019. URL : http:// journals.openedition.org/add/753 ; DOI : 10.4000/add.753

Presses universitaires de Rouen et du Havre 
parties de la mémoire de l'autre pour parvenir ensemble, au prix d'un dialogue interculturel, à un véritable échange qui ne s'apparentera pas uniquement à une perte, pour l'un, des collections anatomiques ou à une revanche, pour l'autre, sur l'ancien système colonial.

Doctorant et chargé d'enseignement en droit public université de Paris I - Panthéon-Sorbonne 



\title{
Jugements de valeur et argumentation juridique
}

\author{
Ángeles Ródenas Calatayud
}

Dans ce texte, j'entends défendre l'opportunité et la viabilité d'une théorie de l'argumentation juridique qui développe un discours systématique, cohérent et fructueux sur le maniement des jugements de valeur de la part des organes d'application dans un contexte d'indétermination du droit. Il s'agit d'une théorie de l'argumentation juridique visant à identifier les principaux contextes argumentatifs dans lesquels les jugements de valeur sont employés par les juges et les autres organes d'application du droit, en offrant une cartographie des situations d'indétermination dans lesquelles les juges se voient obligés de recourir à des considérations fondées sur des jugements de valeur pour résoudre les affaires qui se présentent à eux. En prenant pour point de départ cette cartographie des situations d'indétermination, la théorie de l'argumentation juridique que je propose entend par ailleurs anticiper les stratégies argumentatives relativement stables à partir desquelles, en fonction des caractéristiques de chacun de ces contextes, il est possible d'affronter la tâche argumentative.

Mais avant de se pencher sur ces questions, il convient de réfléchir - ne serait-ce que brièvement - sur le peu d'intérêt que la théorie juridique témoigne à l'égard de ces contextes argumentatifs. À de rares exceptions près, l'analyse des contextes argumentatifs dans lesquels les juges se voient obligés de recourir à des considérations fondées sur des jugements de valeur a constitué une carence récurrente des études de théorie du droit : celle-ci laisse les juristes dépourvus d'outils conceptuels et théoriques à travers lesquels faire face aux défis que présente ce travail. Cette carence n'est pas le produit du hasard, mais résulte d'une stratégie délibérée (quoique pas nécessairement explicitée). Dans la théorie du droit, s'est imposé ce que l'on pourrait appeler une stratégie de l'autruche: on se cache la tête sous l'aile, en opérant une réduction drastique de la réalité qui est l'objet d'étude, et en faisant la sourde oreille vis-à-vis des raisonnements juridictionnels dans lesquels interviennent des considérations morales. 
La cause de ce défaut d'attention, cependant, n'a pas toujours été la même. Nous pourrions identifier la cause la plus commune dans les origines du modèle actuel de théorie du droit positiviste. Il s'agit du souci, d'origine kelsénienne, de la préservation de la pureté de la méthode théorique, associé à un scepticisme marqué en matière morale. Ce souci et ce scepticisme, qui ont été les traits caractéristiques du positivisme juridique, ont laissé leur marque et accompagnent encore, à des degrés et selon des combinaisons variables, une bonne partie de la théorie du droit qui existe aujourd'hui. Logiquement, si de telles précautions sont prises, notre voyage ne nous conduit pas bien loin: nous ne parcourons que les paysages dans lesquels règnent la lumière et la clarté et, dès que la première ombre les approche, nous nous orientons ailleurs. Plutôt que d'assumer le risque de contamination de la théorie du droit par les spéculations qui sont supposées inaccessibles au discours rationnel, se trouve exclue du champ de la réflexion l'analyse du rôle des jugements de valeur dans les délibérations pratiques des organes d'application du droit ${ }^{1}$.

L'autre cause du désintérêt de la théorie du droit pour ces contextes argumentatifs apparait relativement distincte de la précédente, bien qu'elle puisse se combiner avec elle: il s'agit de l'adoption d'une thèse nettement fondée sur des critères liés à l'autorité vis-à-vis de la nature du droit. Selon cette perspective, les ordres de l'autorité établissent pour leurs destinataires des raisons elles-mêmes dotées d'autorité pour réaliser ce à quoi ils les obligent. Par conséquent, on soutient que cette caractéristique du droit serait perdue si nous habilitions les organes chargés de l'application du droit à prendre en compte des considérations fondées sur des jugements de valeur au moment où ils résolvent les cas qui leur sont soumis. Tel est clairement le point de vue adoptée par Joseph Raz qui, paradoxalement, est loin d'être un sceptique sur le plan moral, mais qui, cependant, considère que l'argumentation relative aux valeurs doit demeurer exclue du discours juridique.

Quelle que soit la cause de l'adoption de la stratégie négationniste, la conséquence demeure identique: une lacune de la théorie du droit. Celle-ci ne s'avère pas purement théorique, mais également pratique: il s'agit d'une lacune théorique en ce qu'elle laisse dans l'ombre une partie significative de l'activité que réalisent les juges et les autres organes

1. Tel est clairement le cas de Kelsen et de quelques auteurs contemporains qui ont contribué de manière importante au développement et au perfectionnement de la théorie juridique actuelle, tels que Carlos Alchourrón et Eugenio Bulygin, Riccardo Guastini, Paolo Comanducci, Ricardo Guibourg, etc. 
d'application du droit; il s'agit également d'une lacune pratique en ce que la théorie devient impuissante à fournir des outils conceptuels aux organes d'application du droit lorsque ceux-ci se voient obligés de recourir à des considérations fondées sur des jugements de valeur.

Dans les paragraphes qui suivent, j'entends présenter les fondements d'une proposition théorique destinée à remédier à cette carence traditionnelle de la théorie du droit. Ma stratégie argumentative se développera en deux temps ${ }^{2}$. Je vais d'abord tenter d'identifier le problème auquel nous faisons face, en proposant un catalogue assez complet des situations dans lesquelles les organes d'application du droit sont obligés à recourir à des considérations fondées sur des jugements de valeur (1). Une fois cette cartographie de situations esquissée, je tenterai d'exposer les fondements d'un modèle méthodologique alternatif, à partir duquel il soit possible de construire des stratégies argumentatives prédéfinies pour chacune des situations préalablement identifiées (2).

\section{Cartographie des situations d'indétermination}

Je vais soutenir la thèse selon laquelle, dans des circonstances déterminées, le droit exige des organes d'application du droit qu'ils prennent en considération certains jugements de valeur pour l'identification du droit applicable. Dans de telles situations, le recours aux jugements de valeur ne contreviendrait pas à ce que le droit requiert des juges et autres organes chargés d'appliquer des normes, mais serait, bien au contraire, exigé par celui-ci.

Plus concrètement, je soutiendrai que les jugements de valeur opèrent au moment de l'application du droit dans un double sens. Ils conduisent d'une part à ce que des normes qui, selon les critères liés à l'autorité déterminés dans le système des sources ${ }^{3}$, ne pourraient être identifiés comme du droit, s'avèrent, néanmoins, applicables et, d'autre part, à ce que des normes identifiées comme juridiques selon les critères liés à l'autorité s'avèrent, malgré tout, inapplicables.

Alors que dans le premier cas nous dirions que les jugements de valeur permettent d'identifier comme juridiques des normes qui, selon les critères établis par voie d'autorité dans le système des sources, ne le seraient pas, dans le second nous pouvons affirmer que les jugements

2. Les idées qui suivent sont extraites d'un travail publié antérieurement dans la revue Doxa. Voir Ángeles Ródenas, «¿Qué queda del positivismo jurídico?», Doxa. Cuadernos de Filosofía del Derecho, $\mathrm{n}^{\circ}$ 26, 2006, p. 417-448.

3. En d'autres termes, les critères qui nous permettent d'affirmer que la norme n'appartient au système $S$, sans qu'il soit nécessaire d'en évaluer le contenu. 
de valeur excluent du champ juridique des normes qui, selon ces seuls critères, seraient bien du droit.

En premier lieu, ainsi que je viens de l'indiquer, il existe des normes qui, selon les critères liés à l'autorité fixés par le système des sources, ne seraient pas identifiables comme du droit, et qui s'avèrent, malgré tout, applicables en raison de considérations évaluatives. Dans ces hypothèses, nous pouvons affirmer qu'afin de combler les insuffisances propres d'un système qui opère au seul moyen des normes de commandement, le droit formule un appel pour que la morale (ou d'autres considérations évaluatives) soit incorporée à son propre contenu. En d'autres termes, le droit ouvre la voie, pour la résolution de litiges, à l'utilisation de critères qui ne sont pas fondés sur son système de sources, mais sur des considérations reposant sur des jugements de valeur.

Ainsi, dans de nombreux cas, c'est le législateur lui-même qui prévoit un renvoi à des critères extrajuridiques, fondés sur des valeurs, pour définir le contenu et/ou les conditions d'application de normes déterminées. Certains concepts évaluatifs liés au constitutionnalisme moderne, comme le "traitement dégradant" si souvent mentionné, en représentent de bonnes illustrations. Ce qui est caractéristique de l'utilisation de ce type de concepts tient à ce qu'ils impliquent une renonciation du législateur à introduire des propriétés descriptives dans la norme et, à leur place, un renvoi aux conventions interprétatives en vigueur dans une collectivité sociale déterminée. Ceci ne signifie pas, naturellement, que ces conventions prévoient une réponse pour chacun des cas qui se présentent, mais il existe toujours un ensemble de cas qui s'avèrent paradigmatiques de l'application du concept ${ }^{4}$.

L'inclusion dans le droit de ce type de concepts évaluatifs n'est pas un phénomène nouveau, lié à l'évolution moderne du constitutionnalisme. $S$ 'il est certain que dans le constitutionnalisme moderne cette tendance s'avère plus perceptible, l'inclusion de concepts comme ceux de «bonne foi », «diligence d'un bon père de famille », «honneur », etc., s'avère une caractéristique des systèmes juridiques traditionnels ${ }^{5}$. Dans ces exemples et les exemples similaires, le législateur ouvre consciemment une porte d'entrée à la morale dans le droit ${ }^{6}$, renonçant à réguler les cas

4. Voir José Juan Moreso, «En defensa del positivismo jurídico inclusivo », dans Pablo E. Navarro, María Cristina Redondo (dir.), La relevancia del Derecho. Ensayos de filosofía jurídica, moral y política, Barcelone, Gedisa, 2002, p. 101.

5. Ibid., p. 106.

6. Sur l'inclusion progressive d'aspects moraux dans le droit, voir Francisco Laporta, Entre el Derecho y la Moral, Mexico, Fontamara, 1993, p. 60-63. 
au moyen de propriétés descriptives, et imposant à l'organe d'application du droit de clarifier celles-ci à partir de considérations fondées sur des jugements de valeur.

Mais à l'encontre de ce qu'il pourrait sembler, le renvoi par le législateur à des critères extrajuridiques n'est pas l'unique mécanisme qui permet aux acteurs juridiques d'appliquer des normes qui ne sont pas identifiables selon les critères liés à l'autorité. Il existe par ailleurs d'autres mécanismes qui peuvent être considérés comme une réponse de la «méthode juridique »- ou, si l'on préfère, selon une terminologie plus actuelle dans la théorie du droit, de nos "pratiques juridiques interprétatives » - aux insuffisances d'un système qui opère uniquement à travers des règles impératives. Des figures telles que l'analogia legis prévue dans notre Code civil, ou des constructions doctrinales telles que celle de l'interprétation extensive y trouvent précisément leur point d'ancrage.

En prenant l'interprétation extensive comme exemple, on se rappellera comment la dogmatique soutient que ce type d'interprétation se produit lorsque l'organe d'application du droit étend la formulation littérale d'une norme, pour la mettre en accord avec sa ratio. L'interprétation extensive - dit-on - se produit lorsqu'une norme exprime moins que ce qu'elle souhaite'.

À titre d'exemple d'interprétation extensive, Diez-Picazo et Gullón citent «le cas de l'ancien article 317 (323 actuel), dans lequel était limitée la capacité d'action du mineur émancipé, malgré le fait qu'il était considéré comme une personne majeure pour régir sa personne et ses biens. Le mineur émancipé ne peut, selon ce précepte, ni constituer une sûreté, ni vendre des biens par lui-même. Le sens de la norme consistait à lui interdire de disposer de biens immobiliers, en conséquence de quoi tout acte de disposition, et non seulement la vente, lui était interdit: l'échange d'un tel bien, sa donation, sa dation en paiement d'une dette, la transmission de la propriété en échange de quoi l'acquéreur paierait une rente à vie, etc. Dans soutes ces hypothèses, se produit une sortie du patrimoine de l'émancipé, tout comme en cas de vente. La loi, voit-on, exprimait moins que ce qu'elle voulait ${ }^{8} »$.

Dans cet exemple, nous voyons comment un ensemble de normes qui prohibent une série d'actes de disposition des biens d'un mineur - échange, donation, etc. - s'avèrent applicables, en dépit du fait que

7. Luis Diez-Picazo et Antonio Gullón, Instituciones de Derecho Civil, Madrid, Tecnos, 1998, vol. I/1, p. 101 et suiv.

8. Ibid., p. 102, italiques ajoutées. 
ces actes n'étaient pas visés par la norme prohibitive originelle, qui se référait uniquement à la vente et à la constitution d'une sûreté. Selon la doctrine, ce que le droit requiert de l'autorité d'application n'est pas une application mécanique de la norme, mais au contraire qu'elle réalise un jugement fondé sur des considérations évaluatives qui lui permettent d'étendre les raisons sous-jacentes à la norme à d'autres situations.

En somme, les systèmes juridiques souffrent d'insuffisances qui sont dans une large mesure - ainsi que le soulignait déjà Hart, la conséquence inévitable de la texture ouverte du langage et de la relative indétermination des faits futurs ${ }^{9}$. Mais le droit établit par ailleurs des mécanismes qui permettent de remédier à de telles insuffisances, en recourant à des considérations évaluatives. De tels mécanismes peuvent être prévus par le législateur ou trouver leur origine dans les pratiques juridiques. Ils permettent que des normes qui, en principe, n'appartiennent pas au système, s'avèrent finalement applicables. Les considérations évaluatives qui sont introduites en vertu de tels mécanismes nous permettent de déterminer quelles sont les conditions d'application d'une norme ou bien d'étendre les raisons sous-jacentes à une norme, ou au droit, au-delà de ce que leur teneur littérale permet.

Ainsi qu'on le signalait auparavant, il est également possible que des considérations fondées sur des jugements de valeur conduisent à ne pas appliquer des normes qui, en principe, en ne prenant en compte que les critères liés à l'autorité fixés par le système des sources, seraient du droit. Il me semble intéressant de souligner que, bien que nous puissions rencontrer des cas qui rentrent simultanément dans cette catégorie et celle à laquelle je viens de faire référence - les cas dans lesquels une norme non identifiable selon les critères qui font autorité se trouve, malgré tout, applicable - cette seconde catégorie est conceptuellement indépendante de la précédente ${ }^{10}$. Ce qui définit ces cas tient à ce que les considérations fondées sur des jugements de valeur conduisent à exclure l'application de normes qui, en principe, appartiennent au système. En d'autres termes, alors que dans le premier cas les considérations évaluatives opèrent comme condition suffisante de la juridicité (bien que toujours avec un caractère subsidiaire - en l'absence de réponse fondée

9. Voir H. L. A. Hart, The Concept of Law, Oxford, Clarendon Press, 1961.

10. Tel est ce qui se produit avec les normes qui se réfèrent à l'abus de droit, à la fraude à la loi ou au détournement de pouvoir. Bien qu'il soit clair que, concernant ces normes, la condition d'application est indéterminée, nous allons voir qu'il s'agit aussi de mécanismes que le législateur incorpore pour porter exception à l'application d'autres normes. C'est pourquoi j'ai préféré y faire référence dans ce second paragraphe. 
sur les critères faisant autorité déterminés par les sources), dans le second cas les considérations évaluatives seraient une condition nécessaire de la juridicité, dans la mesure où elles permettent de porter exception au droit fondé sur les critères faisant autorité reconnus dans les sources, voire de l'invalider ${ }^{11}$.

Les systèmes juridiques opèrent fondamentalement à travers des règles de commandement. Ces règles sont le résultat d'une pondération entre les raisons sous-jacentes favorables et hostiles à la réalisation de l'action prescrite. De ce fait, si se produisent les conditions d'application prévues dans la norme, la règle doit être applicable. Cependant, nous savons également qu'aucun système ne peut opérer de manière cohérente en n'utilisant que des normes impératives, de sorte que les systèmes juridiques prévoient des mécanismes, fondés sur des critères évaluatifs, qui permettent d'exclure les normes applicables, soit en introduisant des exceptions au droit fondé sur les sources, soit en l'invalidant ${ }^{12}$.

À nouveau, un regard superficiel sur notre système juridique suffit pour rencontrer des exemples dans lesquels le législateur lui-même prévoit des mécanismes pour porter exception à des règles, permissives aussi bien que prohibitives: l'abus de droit, la fraude à la loi, le détournement de pouvoir sont des exemples clairs d'institutions qui permettent d'écarter des règles permissives. Selon M. Atienza et J. Ruiz Manero, ces trois institutions, qui forment la catégorie générale des illicites atypiques, partagent les caractéristiques suivantes:

1. l'existence, prima facie, d'une action permise par une règle;

2. la production d'un dommage comme conséquence, intentionnelle ou non, de cette action;

3. le caractère injustifié de ce dommage à la lumière de considérations fondées sur un jugement de valeur;

4. la production, à partir de cette pondération, d'une nouvelle règle qui limite le champ d'application de la première, en qualifiant de prohibés des comportements qui, selon celle-ci, apparaissaient permis $^{13}$.

11. Juan Carlos Bayón opère cette distinction pour différencier deux formes possibles de positivisme inclusif, dans "Derecho, convencionalismo y controversia ", op. cit., p. 71-72.

12. L'idée d'exclure les normes juridiques, comme on le verra, demeure en étroite relation avec la notion de dérogeabilité (derrotabilidad) des normes juridiques.

13. Voir Manuel Atienza et Juan Ruiz Manero, Ilícitos atípicos, Madrid, Trotta, 2000. Suivant l'analyse de ces auteurs, la différence entre ces trois institutions serait la suivante: 1) L'abus de droit présuppose uniquement l'existence d'une 
Mais notre système comporte de plus traditionnellement des mécanismes correcteurs pour les règles qui établissent des prohibitions, dont les causes d'irresponsabilité en droit pénal ou l'objection de conscience sont une bonne illustration. Toutes ces institutions remplissent une fonction commune dans notre ordre juridique : porter exception à des règles qui établissent des obligations. Pour qu'une telle opération ait lieu, il est nécessaire que l'autorité d'application du droit réalise une évaluation quant à savoir si, étant données les circonstances du cas, les raisons sous-jacentes aux règles s'appliquent ou non. Ainsi, par exemple, avant d'appliquer la norme qui justifie la légitime défense, l'interprète doit réaliser un raisonnement préalable afin de déterminer si le bien protégé est ou non de valeur égale à celui qui est sacrifié ${ }^{14}$.

Mais le législateur n'est pas le seul à prévoir des mécanismes qui permettent d'exclure des normes applicables sur le fondement de considérations évaluatives. Ainsi qu'il en allait dans le cas inverse - celui des normes non identifiables à travers des critères dotés d'autorité, mais qui s'avéraient néanmoins applicables -, il convient de remarquer que nos pratiques juridiques ont également quelque chose à dire à ce sujet. Ainsi, la dogmatique civiliste espagnole parle sans ambages d'une interprétation restrictive qui serait le résultat d'une limitation du contenu littéral d'une norme pour la rendre conforme à son «contenu substantiel» ou sa « ratio ${ }^{15} »$. Si, comme nous l'avons vu, dans l'interprétation extensive la dogmatique soutient que «la norme dit moins qu'elle ne veut», dans ces cas, elle considère que « la norme dit plus qu'elle ne veut».

«Un exemple d'interprétation restrictive, indiquent Diez-Picazo et Gullón, est celui de l'article 1459.2 du Code civil, qui interdit aux

règle permissive, par exemple l'abus du droit de propriété. 2) Dans la fraude à la loi et le détournement de pouvoir, ce qui est prima facie couvert par une règle permissive est l'utilisation du pouvoir conféré par une norme d'habilitation (un type de norme qui établit que si, dans des circonstances déterminées, un agent réalise une action déterminée, se produit alors un résultat institutionnel ou un changement normatif: un contrat, un acte administratif, une loi, etc.) Il s'ensuit qu'une norme permissive n'intervient pas seule, mais uniquement en conjonction avec une norme habilitante (dont l'utilisation est régulée par la norme permissive). 3) La différence entre les deux dernières réside en ce que, lorsqu'il s'agit de pouvoirs normatifs privés, l'institution pertinente est la fraude à la loi (par exemple utiliser le pouvoir de faire des donations pour transférer un patrimoine au détriment des héritiers); et que lorsqu'il s'agit de pouvoirs normatifs publics, l'institution pertinente est le détournement de pouvoir (par exemple utiliser le pouvoir en matière d'urbanisme uniquement pour favoriser les intérêts d'une entreprise immobilière) [ibid., p. 126-127].

14. Voir José Juan Moreso, art. cité, p. 106.

15. Luis Diez-Picazo et Antonio Gullón, op. cit., p. 101. 
mandataires de se porter acquéreurs des biens de la vente ou de l'administration desquels ils étaient chargés. Puisque le fondement de l'interdiction est le souci de veiller aux intérêts du mandant, si celui qui était chargé de les vendre les acquérait pour lui-même, il pourrait méconnaître ces intérêts; au contraire, lorsque c'est le propriétaire luimême qui vend au mandataire, la prohibition ne trouve pas à s'appliquer, puisque le danger qu'il s'agit de conjurer disparait. La loi dit ici plus que ce qui est souhaité ${ }^{16}$.»

Il s'ensuit qu'une conduite qui est clairement incluse dans le présupposé factuel d'une norme prohibitive - que le mandataire achète ses biens au mandant - s'avère, malgré tout, permise au regard des raisons mêmes qui servent de justification à cette norme - la protection des intérêts du mandant.

À ces institutions, qui démontrent la présence dans le droit, depuis longtemps, de mécanismes prévus par le législateur ou par nos pratiques interprétatives pour porter exception à des normes applicables en raison de considérations évaluatives, s'ajoutent les prévisions législatives caractéristiques du constitutionnalisme moderne. Elles permettent, que ce soit aux organes juridictionnels ordinaires ou aux tribunaux constitutionnels, d'invalider des normes dont le contenu méconnait les valeurs fondamentales de la constitution.

En somme, et à titre de conclusion de ce que nous avons vu dans ce paragraphe, nous pourrions dire que, dans certaines occasions, le droit requiert des organes d'application qu'ils prennent en compte certaines considérations évaluatives comme condition suffisante ou nécessaire de la juridicité.

Dans le tableau page suivante, je tente de résumer l'ensemble des situations auxquelles je me suis référée dans ce paragraphe.

\section{Une proposition méthodologique alternative}

Parvenu à ce point, il est nécessaire de formuler les bases d'une proposition méthodologique alternative, à partir de laquelle il soit possible de reconstruire la tâche argumentative qu'accomplissent les organes d'application du droit lorsqu'ils font face à des cas comme ceux qui précèdent. Cette proposition repose sur deux piliers: en premier lieu, une compréhension adéquate des raisons que le droit incorpore, en distinguant entre règles, valeurs et compromis entre valeurs (2.1)

16. Ibid., p. 102. 


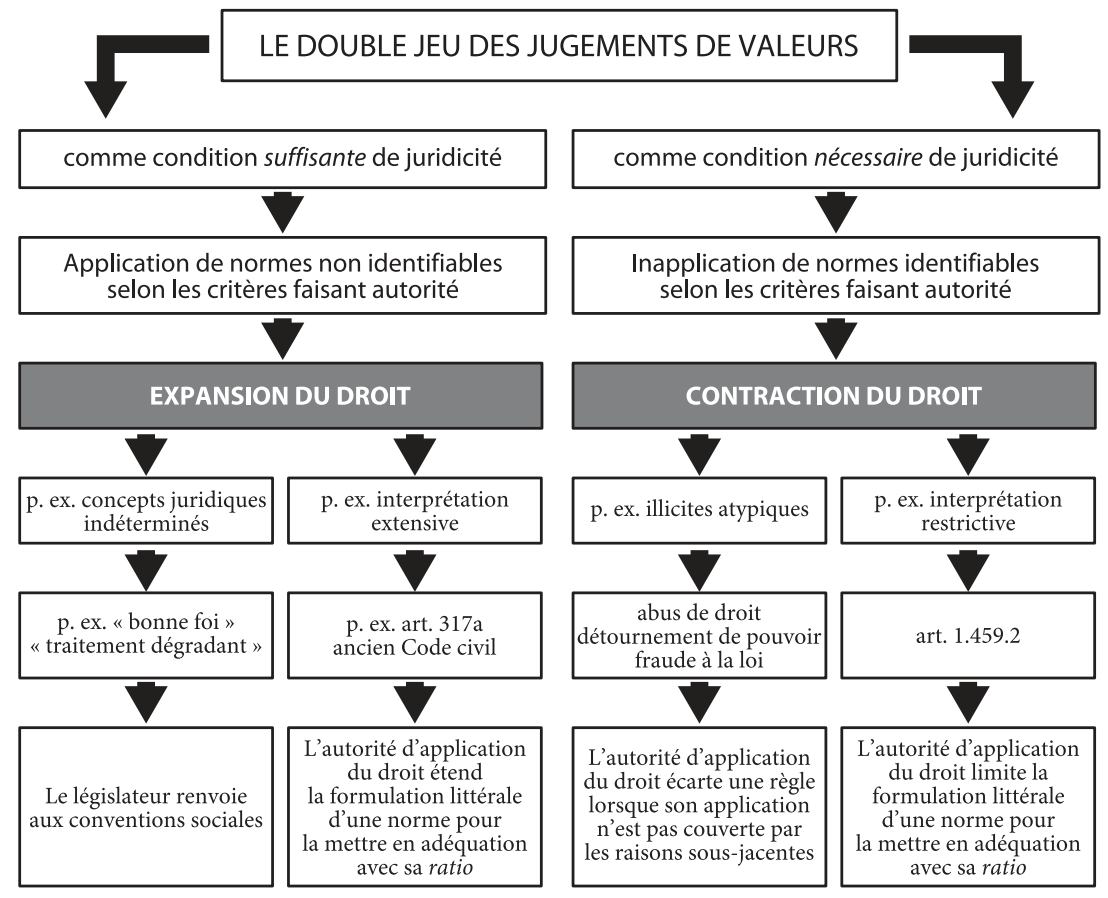

Le double jeu des jugements de valeur.

et, en second lieu, une compréhension adéquate du rôle que jouent les conventions interprétatives et l'idée de cohérence dans l'identification du droit ${ }^{17}$ (2.2).

\subsection{Règles, valeurs et compromis en droit}

Bien entendu, le droit ne se compose pas uniquement de règles dotées d'une autonomie sémantique, mais également de valeurs. Les ordres juridiques incorporent un large catalogue de valeurs dans leurs chartes constitutives, mais le droit ne se limite pas seulement à reconnaître des valeurs. Fréquemment, il établit également des priorités entre elles, que

17. Je ne soutiens pas que le droit ait toujours une réponse pour tous les cas. J'admets qu'il est possible que le droit n'ait pas de réponse dans les cas où il est impossible d'accéder aux formes sociales partagées, parce qu'elles n'existent pas. Mais cette question présente des difficultés spéciales, qui justifient que je ne l'aborde pas présentement. 
ce soit vis-à-vis d'un secteur ou d'un sous-secteur de l'ordre juridique ${ }^{18}$ ou vis-à-vis de cas plus ou moins génériques ${ }^{19}$. Ce phénomène s'avère très perceptible lorsque nous explorons les raisons sous-jacentes aux règles impératives ${ }^{20}$. C'est précisément en termes de pondération des valeurs ou des raisons opposées pour des cas généraux que nous prenons conscience des justifications sous-jacentes des règles impératives ${ }^{21}$.

Nous prenons comme point de départ un exemple dont je me suis servi dans un travail antérieur: un signal graphique qui prohibe les appareils radiophoniques dans la bibliothèque universitaire. L'indication pourrait avoir été fondée sur la gêne qu'occasionne aux usagers de la bibliothèque le bruit de ces appareils. Le jugement selon lequel une telle raison vient à prévaloir sur celle que constitue le désir de certains usagers d'écouter la radio à la bibliothèque représente la justification sous-jacente de la règle.

Or, si nous nous intéressons au signal, tout ce qu'il nous dit est qu'il est interdit d'introduire des appareils radiophoniques dans la bibliothèque. Nous sommes capables d'attribuer une signification au signal qui interdit les appareils radiophoniques dans la bibliothèque, bien que nous ignorions quelle est la justification sous-jacente de cette disposition. Il en résulte que nous pouvons affirmer que les formulations normatives - par exemple « Les appareils radiophoniques sont interdits dans la bibliothèque » - possèdent une autonomie sémantique, c'està-dire que leur signification - ce qu'elles prescrivent - peut être établie sans prendre en compte la justification qui les sous-tend. Selon Frederick Schauer, nous pouvons comprendre les prescriptions contenues dans les formulations normatives comme des généralisations qui tentent d'illustrer le résultat que, normalement, nous pourrions obtenir par

18. Par exemple, tel est ce qui se produit en droit pénal, où le principe de légalité acquiert un poids décisif qu'il ne revêt pas dans d'autres secteurs de l'ordre juridique, ou avec le principe d'autonomie de la volonté en relation avec les obligations et les contrats en droit civil.

19. La prévalence entre raisons ne peut guère être absolue, sauf à ce qu'il faille faire référence à des classes de cas plus ou moins génériques dans lesquels sont précisées les conditions d'application dans lesquelles une collision entre normes est prévue.

20. J'ai abordé ces questions de manière détaillée dans Razonamiento judicial y reglas, Mexico D. F., Fontamara, 2000 et dans «En la penumbra: indeterminación, derrotabilidad y aplicación judicial de normas ", Doxa. Cuadernos de Filosofía del Derecho, $\mathrm{n}^{\circ} 24,2001$.

21. Évidemment, ces considérations ne s'appliquent pas aux règles impératives qui résolvent des problèmes de pure coordination, par exemple conduire à droite ou à gauche. Mais ce type de règles ne me semble pas constituer l'exemple central de la règle impérative. 
l'application directe des justifications ${ }^{22}$ (ne pas gêner les usagers de la bibliothèque). Comme nous venons de l'indiquer, cette distinction entre la signification de ces formulations normatives et la justification sous-jacente de celles-ci nous offre un bon instrument d'analyse à partir duquel aborder les cas dont je me suis occupée dans le paragraphe précédent.

Bien qu'il soit caractéristique pour le droit de s'exprimer à travers des formulations normatives qui possèdent une autonomie sémantique - et il existe de puissantes raisons pour qu'il en soit ainsi -, tel n'est pas toujours le cas. Parfois, le droit se limite à montrer quelles sont ses valeurs sous-jacentes, et laisse aux autorités d'application le soin de déterminer si ces valeurs s'appliquent ou non dans un cas concret. Tel est ce qui se produit lorsque le législateur emploie des concepts évaluatifs. Comme nous l'avons vu précédemment, dans ces hypothèses, le droit renonce à réguler les cas à travers des propriétés descriptives, et invite le juge à déterminer ces propriétés. Nous ne pouvons pas pour autant taxer la fonction d'application du juge de purement discrétionnaire. En d'autres termes, je ne crois pas que dans ces cas le contenu de la solution édictée soit indifférent pour le droit. Les concepts évaluatifs combinent des cas clairs, dans lesquels, à la lumière de nos pratiques interprétatives, il n'est pas discutable que la subsomption d'un cas individuel sous le concept évaluatif s'opère ${ }^{23}$, et des cas difficiles, dans lesquels diverses conceptions d'un même concept conduisant à des solutions différentes sont en concurrence ${ }^{24}$. Lorsqu'il s'agit d'un cas clair, il me parait indiscutable que l'interprète est guidé par la signification conventionnelle du concept. Mais je crois que, même dans un cas difficile, il est défendable d'affirmer que la solution à adopter n'est pas indifférente au droit. Tout dépend de la conception de nos pratiques interprétatives que nous retenons.

Selon Bayón ${ }^{25}$, nous pouvons distinguer deux manières de comprendre le conventionnalisme. Pour la première d'entre elles - que Bayón nomme «conventionnalisme superficiel» - les critères de correction d'un concept vont jusqu'à ce qu'un accord explicite de la communauté de référence sur l'ensemble de ses applications correctes soit

22. Voir Frederick Schauer, Playing by the Rules, Oxford, Clarendon Press, 1991.

23. Il existe des centaines d'exemples de cette hypothèse qui, en accord avec nos pratiques interprétatives, appelleraient sans hésitation la qualification de traitement dégradant.

24. Voir José Juan Moreso, art. cité, p. 100-101.

25. Voir Juan Carlos Bayón, art. cité, p. 78-81. 
établi. De ce fait, au-delà de l'extension de cet accord explicite, il est impossible de parler - par définition - de critères partagés. Au contraire, pour l'autre manière de concevoir le conventionnalisme - que Bayón nomme «conventionnalisme profond "-, il est possible d'affirmer que, dans un certain sens, les conventions existent, quand bien même il y existerait des désaccords quant à leur contenu. S'inspirant de Raz, Bayón soutient que l'identification de cas paradigmatiques de la part d'une communauté implique de maitriser une technique d'utilisation, mais que ceci ne requiert pas davantage qu'une connaissance tacite des critères de correction, puisque ce n'est pas l'accord explicite au sujet des applications concrètes qui définit ces pratiques comme correctes, mais l'arrière-plan de critères partagés. En toute hypothèse, l'objectivité des faits dont cette conception prétend rendre compte s'appuie sur un ensemble de croyances et d'attitudes partagées, de sorte que la convention puisse être encore considérée comme conventionnaliste, bien que pour ce type de conventionnalisme les critères de correction d'un concept ne coïncident pas exactement avec ce que la communauté soutient explicitement à ce sujet.

Envisager les concepts évaluatifs à partir des postulats du conventionnalisme profond suppose d'affirmer que, même lorsque nous rencontrons un cas situé dans une zone de pénombre, l'affirmation selon laquelle l'acteur juridique n'a pas un pouvoir discrétionnaire conserve du sens, dans la mesure où, au-delà des accords explicites relatifs aux applications concrètes d'un concept, des critères tacites de correction de ceux-ci sont sous-jacents. Il s'ensuit qu'il est opportun d'appliquer aux concepts évaluatifs une stratégie de type rawlsien, selon laquelle ces concepts doivent être modelés ${ }^{26}$. S'impose de ce fait une méthodologie cohérentiste qui exigerait de partir des cas clairs pour formuler une hypothèse quant aux conditions d'application des concepts, en tentant de confronter cette hypothèse à nos intuitions les mieux établies, de sorte que, si ce test échoue, soit ces intuitions doivent être abandonnées, soit l'hypothèse doit être reformulée. Adopter ce point de vue n'implique pas, naturellement, de supposer que pour tous les cas de désaccord il soit possible de trouver une réponse correcte. Tel n'est le cas que s'il existe un arrière-plan de critères partagés - qui ne doivent pas nécessairement être parfaitement déterminés à première vue - mais au-delà de telles conventions, nous rencontrons les limites du droit ${ }^{27}$.

26. Voir José Juan Moreso, art. cité, p. 100.

27. Dans le même sens, voir Juan Carlos Bayón, art. cité, p. 81. 
Revenant sur la distinction entre la signification des formulations normatives et leur justification sous-jacente, je vais l'approfondir quelque peu pour obtenir des critères qui permettent de rendre compte des autres cas, que je range sous la rubrique des «tensions internes du droit». Dans certains cas, un défaut d'ajustement entre ce que la formulation normative exige de nous et ce que sa justification sous-jacente requiert peut se produire. Ce type d'expérience est connu sous le nom d'" expérience récalcitrante", et peut revêtir deux formes. Dans certains cas particuliers, la généralisation contenue dans la formulation normative n'inclut pas certains états de choses qui peuvent contribuer à la conséquence représentée dans la justification sous-jacente à la règle, c'està-dire lorsque les prescriptions contenues dans les formulations normatives n'incluent pas certains présupposés auxquels, au contraire, leur justification sous-jacente serait applicable. Par exemple, l'interdiction des appareils radiophoniques dans la bibliothèque ne concerne pas les baladeurs, ni les téléviseurs, etc. Mais il parait clair que leur utilisation causerait une gêne semblable aux usagers de la bibliothèque. Dans de tels cas, la prescription contenue dans la formulation normative est infra-incluante par rapport à sa justification sous-jacente, en ce sens que cette prescription n'inclut pas certains faits - baladeurs, téléviseurs, etc. auxquels sa justification serait applicable. En revanche, la prescription contenue dans la formulation normative est supra-incluante lorsqu'elle comprend des états de choses qui ne produisent pas la conséquence représentée dans la justification de la règle. Revenant à l'exemple du signal qui interdit les appareils radiophoniques dans la bibliothèque, il est aisé de concevoir des hypothèses dans lesquelles la justification sousjacente de cette règle ne serait pas applicable: l'employé qui nettoie la bibliothèque avant l'heure d'ouverture au public, la bibliothécaire qui, en cas d'alerte pour cause d'inondations, attend un avis de protection civile pour évacuer la bibliothèque, etc. Dans ces hypothèses, la prescription contenue dans la formulation normative est supra-incluante par rapport à sa justification sous-jacente, au sens où une telle prescription inclut certains présupposés - l'employé du nettoyage ou la bibliothécaire dans lesquels sa justification ne serait pas applicable.

Comme je viens de le mentionner, ces deux formes d'expériences récalcitrantes offrent un bon instrument d'analyse à partir duquel aborder le reste des cas que j'ai identifiés. Ainsi, dans les pages précédentes, j'ai indiqué, en premier lieu, que des considérations fondées sur des jugements de valeur permettent d'identifier comme juridiques des normes qui, selon les critères dotés d'autorité du système des sources, ne le seraient pas. L'interprétation extensive constituait un exemple clair avec lequel 
illustrer cette hypothèse, puisque, selon la dogmatique, « la loi dit moins que ce qu'elle veut » et l'autorité d'application du droit se trouve forcée d' «étendre la formulation littérale d'une norme, pour la mettre en accord avec sa ratio ». En recourant aux notions que je viens d'introduire, nous pourrions rendre compte de l'interprétation extensive en termes de réponse de l'autorité d'application du droit à une expérience récalcitrante causée par le caractère infra-incluant d'une formulation normative; les prescriptions contenues dans les formulations normatives n'incluent pas certains présupposés auxquels, cependant, leur justification sous-jacente est applicable. Dans l'exemple présenté, la prescription contenue dans la formulation normative - l'interdiction au mineur de constituer une sûreté ou de vendre les biens par lui-même - est infra-incluante par rapport à sa justification sous-jacente - la protection du patrimoine immobilier du mineur -, en ce sens qu'une telle prescription n'inclut pas certains présupposés - l'échange, la donation, etc. - auxquels serait applicable sa justification.

Admettre que les juges peuvent appliquer des normes qui ne sont pas identifiables selon les critères liés à l'autorité fixés dans le système des sources est une chose; parler de pouvoir discrétionnaire dans ces hypothèses en est une autre. Le droit ne renonce pas à déterminer quand les juges peuvent appliquer des normes qui ne sont pas identifiables selon les critères de l'autorité, ni ne se montre indifférent à quel peut être leur contenu. L'acteur juridique qui applique une norme non identifiable selon les critères établis par voie d'autorité devra développer une argumentation tendant à prouver que, en accord avec le droit, des raisons sont présentes pour résoudre le cas pendant en appliquant telle ou telle norme, et que le contenu de cette norme est cohérent avec les autres valeurs de l'ordre juridique. Bien que nous évoluions à un niveau élevé d'abstraction, il est possible de concrétiser quelque peu le sens de cette argumentation, en distinguant trois niveaux: en premier lieu, les arguments destinés à tenter d'établir quelle est la pondération de raisons qui sous-tend la norme dont on prétend étendre la signification littérale ; en deuxième lieu les arguments visant à souligner la grave omission que supposerait, à la lumière de la pondération des raisons sous-jacentes à la norme, le fait de se cantonner à la teneur littérale de la norme, et enfin les arguments qui montrent la conformité de la nouvelle règle vis-à-vis de la pondération des raisons sous-jacentes à la norme préexistante.

Ensuite, la notion d'expérience récalcitrante due au caractère supraincluant des prescriptions va nous aider à rendre compte des cas dans lesquels, en raison de considérations évaluatives, l'application d'une 
norme identifiable comme juridique selon les critères liés à l'autorité qui figurent dans le système des sources est exclue.

Tous les cas inclus dans cette catégorie partagent le fait que, bien que d'après la signification de la formulation normative il serait possible de subsumer les fais du cas sous la norme, l'application de celle-ci s'avère inadéquate au regard de sa justification sous-jacente. En d'autres termes, la signification de la formulation normative inclut des cas auxquels la justification sous-jacente à la norme ne s'applique pas. Ailleurs, j'ai distingué deux types de présupposés qui coïncident parfaitement avec ce type d'expérience récalcitrante. Il s'agit des présupposés qui demeurent hors du champ d'application d'une règle et de ceux qui constituent une exception à celle-ci ${ }^{28}$. Commençons par les premiers. Un cas se trouve au-delà du champ d'application d'une règle si les principales raisons sur lesquelles la règle s'appuie ne sont pas applicables à ce cas. Si nous revenons à l'exemple de l'interdiction des appareils radiophoniques dans la bibliothèque, le présupposé de l'employé du nettoyage qui allume la radio avant l'heure d'ouverture au public pourrait être considéré comme un cas situé hors du champ d'application de la règle, puisque l'action de l'employé ne cause aucune gêne aux usagers de la bibliothèque. De même, l'exemple qui servait pour illustrer l'interprétation restrictive est une hypothèse claire de cas situé au-delà du champ d'application de la règle : l'article $1459.2 \mathrm{du}$ Code civil interdit aux mandataires l'achat des biens de la vente ou de l'administration desquels ils ont été chargés. La raison sous-jacente de cette prohibition tient aux intérêts du mandant, puisque si la personne chargée de les vendre les acquérait elle-même, elle pourrait violer ces intérêts. Mais, lorsque le propriétaire lui-même vent au mandataire, la dogmatique estime que la prohibition ne s'applique pas. Il s'agit, en somme, conformément à ce que nous venons de voir, d'un cas situé hors du champ d'application de la règle ${ }^{29}$. Au contraire, un cas s'avère une exception à la règle lorsque lui sont applicables certaines des principales raisons qui soutiennent la règle, mais lorsqu'il existe d'autres raisons qui n'ont pas été prises en compte dans la pondération de raisons que la règle a en vue, et dont le poids est décisif pour la résolution de l'affaire. Ainsi, dans le cas de la bibliothécaire qui allume la radio dans l'attente d'une directive de protection civile, nous pourrions

28. Razonamiento judicial y reglas, Mexico D. F., Fontamara, 2000 et «Indeterminación, derrotabilidad y aplicación judicial de normas », op. cit. J’ai développé ces catégories à partir d'une distinction présentée par Raz dans Razón práctica y normas, Madrid, Centro de estudios constitucionales, 1991, p. 82.

29. Luis Diez-Picazo et Antonio Gullón, op. cit., p. 102. 
comprendre que nous sommes en présence d'une exception à la règle, dans la mesure où il est certain que l'action de la bibliothécaire engendre une gêne pour les usagers de la bibliothèque. Ce qui se produit est que l'on considère que l'information qu'elle attend est vitale afin de limiter le risque pour la vie des personnes qui se trouvent dans la bibliothèque et que cette dernière raison, la valeur de la vie humaine, prévaut sur les autres, que vise le compromis (ou le jugement de prévalence) exprimé dans la règle. Dit autrement, ce que la règle vise est simplement que la tranquillité des usagers de la bibliothèque (raison 1) prévale sur le désir de certains usagers d'écouter la radio (raison 2), mais non vis-à-vis de la valeur de la vie humaine (raison 3). En termes d'exception à la règle, nous pouvons rendre compte des autres cas d'inapplication de normes appartenant au système, comme les causes d'irresponsabilité du droit pénal ou l'objection de conscience. Ainsi qu'il apparaît dans les exemples antérieurs, tant dans les hypothèses d'exception aux règles que dans les cas situés en dehors de leur champ d'application, la prescription contenue dans la formulation est mise en question. Mais tel n'est pas le cas de la pondération des raisons sous-jacente à celle-ci. Ceci est dû à ce que les questions relatives au champ d'application et aux exceptions aux règles peuvent être résolues sans rouvrir le débat sur la pondération des raisons sous-jacentes. Le vigile qui n'empêche pas l'employé du nettoyage ou la bibliothécaire d'écouter la radio ne réalise pas nécessairement une pondération avec toutes les raisons de premier ordre applicables au cas. Il peut considérer que le compromis que reflète la règle qui interdit les appareils radiophoniques - la tranquillité des usagers prévaut face au désir d'écouter la radio - est pleinement valide et, de ce fait, demeure sans subir de dérogation. Toutefois, les hypothèses précédentes restent hors du champ d'application de la règle ou constituent une exception. De ce fait, dans ces cas, c'est la prescription contenue dans la formulation normative à travers laquelle la règle s'exprime qui est mise de côté, mais non la pondération des raisons qui lui sont sous-jacentes. En somme, nous pourrions souligner que les juges et les autres autorités d'application du droit peuvent écarter une règle lorsqu'ils estiment que le cas en question se trouve hors du champ d'application de la règle ou représente une exception à celle-ci. Mais, comme j'y insiste, les autorités d'application du droit ne sont pas libres de formuler les exceptions ou les exclusions du champ d'application des normes qu'elles souhaitent, quand elles le souhaitent. En termes très abstraits, les juges devront développer une argumentation tendant à établir, en premier lieu, quelle est la pondération de raisons qui sous-tend la formulation normative dont on prétend restreindre l'application. De 
plus, le juge devrait fournir des arguments qui montrent l'erreur grave en quoi consisterait le fait de ne prêter attention qu'à la signification de la formulation normative, soit parce que le cas discuté constitue une exception à la règle, soit parce qu'il est exclu de son champ d'application. Si le cas ne relève pas du champ d'application de la règle, l'argumentation tendra à montrer que les raisons sous-jacente à la règle dont le poids est décisif pour faire pencher la balance ne trouvent pas à s'appliquer à ce cas. En revanche, si le cas constitue une exception à la règle, le juge devra apporter une autre raison additionnelle - non envisagée dans la pondération des raisons sous-jacentes à la règle - et prouver les éléments suivants: en premier lieu, qu'il s'agit d'une raison qui jouit déjà d'une reconnaissance (expresse ou tacite) dans l'ordre juridique; de plus, que cette raison devrait avoir été prise en compte dans la pondération des raisons applicables au cas en question; enfin, que la nouvelle raison a un poids décisif qui fait définitivement pencher la pondération des raisons applicables au cas en sa faveur. Pour ce dernier aspect, il sera nécessaire de se référer aux cas paradigmatiques qui reposent sur des pondérations entre raisons semblables à celles applicables au cas discuté, et dans lesquels la raison exclue revêt un poids décisif.

Ce que j’ai dit jusqu'ici se réfère aux présupposés dans lesquels, au regard de la pondération des raisons sous-jacentes, on exclut l'application d'une norme identifiable comme juridique en fonction des critères faisant autorité dans le système des sources. Mais il pourrait se produire que, au regard de cette pondération, la norme s'avère invalide. Pour rendre compte de ces hypothèses, je dois revenir sur la distinction entre prescriptions contenues dans la formulation normative des règles et justifications sous-jacentes de ces formulations. Parler de "dérogeabilité » a du sens aux deux niveaux. Ce qui fait l'objet d'une dérogation peut aussi bien être - comme dans les exemples antérieurs - la prescription contenue dans la formulation normative - les radios sont interdites dans la bibliothèque - que les compromis entre raisons sous-jacentes aux règles (ou les jugements de prévalence). Il s'ensuit qu'il est possible de même, ensuite, de manière exceptionnelle, que les juges et les autres autorités d'application du droit laissent inappliquée une règle lorsqu'ils estiment que le compromis entre raisons sous-jacentes à la formulation normative est mal construit. Ceci se produit lorsque, à la lumière d'autres compromis ou jugements de prévalence entre raisons, qui sont implicites ou explicites dans d'autres parties du système juridique et qui sont applicables au cas en question, on se rend compte d'une erreur dans ce compromis lui-même entre les raisons de premier ordre qui opèrent comme justification sous-jacente de la règle. Ce qui est caractéristique de 
cette dérogeabilité au niveau des justifications sous-jacentes aux règles, vis-à-vis de la dérogeabilité au niveau des prescriptions contenues dans les formulations normatives, tient à ce que la règle échoue, dans ce cas comme dans les autres, en tant que raison protégée. Il ne s'agit pas, comme dans les exemples antérieurs, de problèmes résultant du caractère supraincluant de la prescription contenue dans la formulation normative, qui ne mettent en aucun cas en question le jugement de prévalence entre raisons de premier ordre qui opère comme justification sous-jacente de la règle. Ce qui est réfuté comme erroné c'est le jugement de prévalence lui-même qui opère comme raison sous-jacente à la règle, et par lequel on considère que celle-ci ne doit plus jouer le moindre rôle dans la prise de décision future ${ }^{30}$. Ce qui est mis en cause dans le premier type de cas est uniquement l'applicabilité de la prescription au cas concret, tandis que dans le second, il s'agit de sa validité. Alors que dans le premier cas nous disons que la norme est écartée, elle est annulée dans le second. Admettre la dérogeabilité de la justification sous-jacente aux règles ne suppose pas uniquement de nier que le droit détermine positivement la conduite des juges. Nous ne pouvons pas davantage parler de pouvoir discrétionnaire de la part du juge lorsque celui-ci apprécie la dérogeabilité des justifications sous-jacentes aux règles ${ }^{31}$. Pour commencer, un juge ne peut pas rejeter comme erroné le compromis entre raisons contenu dans une norme juridique applicable en se fondant sur des raisons non reconnues jusqu'alors dans l'ordre juridique. Par exemple, il ne peut pas en appeler simplement à la réalisation des intérêts de certains groupes sociaux, à la mise en œuvre de programmes politiques, à la promotion de nouveaux objectifs sociaux ou à la défense de nouvelles valeurs non incorporées jusque-là à l'ordre juridique. Des raisons de ce type ne peuvent être utilisées que par le législateur pour justifier l'approbation d'une règle qui incorpore un nouveau compromis entre raisons de premier ordre, ou l'abrogation d'une règle qui contient

30. Dans Razonamiento judicial y reglas je parlais d'erreurs de premier degré et d'erreurs de second degré pour me désigner à deux types d'erreurs - consécutives aux deux formes de dérogeabilité des règles auxquelles je viens de me référer - que commettaient les juges qui appliquaient ces règles (op. cit., p. 77 et suiv.) Les erreurs de premier degré sont liées à la dérogeabilité au niveau des formulations normatives, tandis que les erreurs de second degré sont liées à la dérogeabilité au niveau des justifications sous-jacentes aux règles.

31. Je suis de près les considérations formulées par Josep Aguiló dans «Independencia e imparcialidad de los jueces y argumentación jurídica », Isonomía, nº 6, avril 1997, p. 75. 
un compromis que l'on estime inadéquat. Mais jamais ceci ne peut être affirmé par les organes d'application.

De plus, les juges ne peuvent pas davantage composer selon leur caprice un nouveau compromis entre les raisons de premier ordre contenues dans les règles. Ce serait une erreur de supposer que l'acteur juridique qui décide d'écarter une règle dispose d'un pouvoir discrétionnaire total pour composer la pondération des raisons qu'il estime opportune, de sorte qu'il le fait en se fondant sur des raisons déjà reconnues par l'ordre juridique: les juges ne peuvent qu'écarter les règles en leur opposant la prévalence d'autres compromis entre les raisons de premier ordre applicables au cas en question, et qui figurent déjà expressément ou tacitement dans le système, ce qui leur impose de développer une argumentation tendant à faire état de cas paradigmatiques dans lesquels les résultats des pondérations indiquent une orientation différente.

En somme, tant dans le cas de la dérogeabilité des prescriptions contenues dans les formulations normatives, que dans celui des justifications sous-jacentes aux règles, pour pouvoir écarter une règle en principe applicable, le juge doit se fonder sur un système de valeurs qui lui est donné par avance et dont il n'est pas le créateur. De ce fait, la solution qu'il prévoit pour le cas en question peut passer le test de la cohérence normative ou de la congruence.

Dans le tableau ci-contre, je tente de résumer la reconstruction proposée dans ce paragraphe.

\subsection{Le recours aux conventions et à la cohérence dans l'identification du droit}

Ces réflexions tentent de montrer comment, à partir de la théorie du droit, il est possible de développer un cadre conceptuel susceptible de rendre compte des mécanismes concrets que, comme nous l'avons vu dans les pages qui précèdent, tant le législateur que la dogmatique juridique ont développés pour pouvoir appliquer les normes qui ne sont pas identifiables selon les critères posés par l'autorité ou pour écarter des normes identifiables par cette voie. Tous les mécanismes que nous avons étudiés obéissent à une même nécessité: doter de cohérence (de cohérence évaluative ou justificative) le système juridique. Il s'agit de faire en sorte d'ajuster la dimension directive et la dimension justificative du droit, ce que l'on nous ordonne et les raisons pour lesquelles on nous l'ordonne. En ce sens, ces catégories ou des catégories semblables sont 